\title{
Flow cytometry detection of reactive plasmacytosis and HRS-like cells as a challenging initial finding in angioimmunoblastic T cell lymphoma
}

\author{
David Azoulay ${ }^{1} \cdot$ Vadim Sonkin $^{2} \cdot$ Luiza Akria $^{1} \cdot$ Ety Shaoul $^{1} \cdot$ Simona Rosen $^{1}$ • \\ Andrei Braester ${ }^{1}$
}

Received: 16 July 2015 / Accepted: 25 January 2016 / Published online: 6 February 2016

(C) Springer-Verlag Berlin Heidelberg 2016

Reactive plasmacytosis is a rare finding that can obscure the underlying etiological disease, leading to misdiagnosis and inappropriate therapy. Here, we report a case of angioimmunoblastic $\mathrm{T}$ cell lymphoma that is represented by a striking amount of reactive plasmacytosis-expressing variable CD138 molecule (Fig. 1).

A 79-year-old female was referred to our medical center due to unexplained fever, fatigue, and weakness. She was found to have lymphadenopathy and Epstein-Barr virus/cytomegalovirus (EBV/CMV)-negative serology. Her CBC showed anemia (Hb $5.7 \mathrm{~g} / \mathrm{dL}$ ), thrombocytopenia $\left(13 \times 10^{3} / \mu \mathrm{L}\right)$, and leukocytosis $\left(41.6 \times 10^{3} / \mu \mathrm{L}\right)$. The leukocyte differential was as follows: neutrophils $55.2 \%$, monocytes $16.2 \%$, lymphocytes $23.4 \%$, eosinophils $4.8 \%$, and basophils $0.4 \%$ (done by Sysmex XN-1000 hematology analyzer). Plasma cells (PCs) with varied morphological appearance were observed in the peripheral blood (PB) smear and raised a suspicion for plasma cell leukemia. Serum electrophoresis shows hypergammaglobulinemia with inconclusive clonality by immunofixation.

Flow cytometry analysis of the PB showed $20 \%$ CD38++ PCs expressing variable CD138 expression. Further immunophenotyping of the PCs showed no cyto-

David Azoulay

David.azoulay@naharia.health.gov.il

1 Hematology Unit, Bar Ilan Faculty of Medicine in the Galilee Nhaharia, Galilee Medical Center, POB 21, 22100 Naharia, Israel

2 Pathology Unit, Bar Ilan Faculty of Medicine in the Galilee Nhaharia, Galilee Medical Center, Naharia, Israel plasmic Ig light chain restriction; positivity for CD45, CD27, and CD19; and negativity to CD56. DNA cell cycle analysis showed S-phase fraction that stained positive with CD38 and not with CD138, suggesting mitotic activity preferentially by the immature plasmablasts. Analysis of bone marrow (BM) aspirate by flow cytometry showed $20 \%$ PCs and $10 \%$ mature B cells, both with no Ig light chain restriction. IGH gene rearrangement that was performed in the BM aspirate showed clonal $D_{\mathrm{H}^{-}} \mathrm{J}_{\mathrm{H}}$ peak with polyclonal background. BM T cells accounted for $8.5 \%$ of total cells and comprised by $87 \%$ CD $4+$ cells and $10 \%$ CD8 cells. Tissue crumbs and cell wash from a core biopsy that was taken from the patient's inguinal lymph node showed $15 \%$ PCs and few B cells, both with no Ig light chain restriction. IGH rearrangement that performed in the nodal specimen determined as polyclonal. $\mathrm{T}$ lymphocytes accounted for $60 \%$ of total cells, comprising of $90 \%$ CD $4+$ cells and $10 \%$ CD 8 cells. In addition, we observed a distinct subset (5\% of total cells) of CD $45+/$ $\mathrm{FSC}^{\text {high }} / \mathrm{CD} 30^{+} / \mathrm{CD} 15^{+/-} / \mathrm{CD} 20^{-} / \mathrm{CD} 138^{-}$cells that were suspected to be Hodgkin-Reed-Sternberg (HRS)-like cells.

Immunohistopathological assessment of the BM confirmed the striking amount of infiltrating PCs which evaluated to be around 30-40\% of the hematopoietic space cellular compartment as detected by CD138. Morphological assessment of the lymph node showed effaced follicular architecture, increased numbers of arborizing high endothelial venules, and atypical lymphocytes with a different size. Immunohistochemical staining showed predominancy of $\mathrm{T}$ cells $(\mathrm{CD} 3+/ \mathrm{CD} 5+)$, and the majority of them were CD4+. In addition, we found the appearance of 3-5\% of HRS-like cells that were characterized by $\mathrm{LCA}+/ \mathrm{CD} 20+/ \mathrm{CD} 30+/ \mathrm{CD} 15^{-}$. The HRS-like cells were negative to EBV. Finally, follicular dendritic 
Fig. 1 AITL related reactive plasmacytosis. a Peripheral blood smear showing plasma cells (PCs) with varied morphological appearance. b Flow cytometry illustration showing BM PCs expressing variable CD138 and c non-restricted cytoplasmic Ig light chains. d Flow cytometry DNA analysis showing S-phase fraction that stains positive with CD38 and not with CD138. The dotted line represents the borderline between negative and positive events. e Flow cytometry illustration of CD45/FSC profile of the CD30+ HRS-like cells (marked in purple) that are observed in cell wash of the biopsy. The events stained with gray color represent the total background cells. f Illustrations from BM biopsy immunohistochemistry showing H\&E (upper) and CD138 (lower) staining. g Illustrations from lymph node biopsy immunohistochemistry showing $\mathrm{H} \& \mathrm{E}$ (upper left), CD3 (lower left), CD30 (upper right), and CD15 (lower right) staining
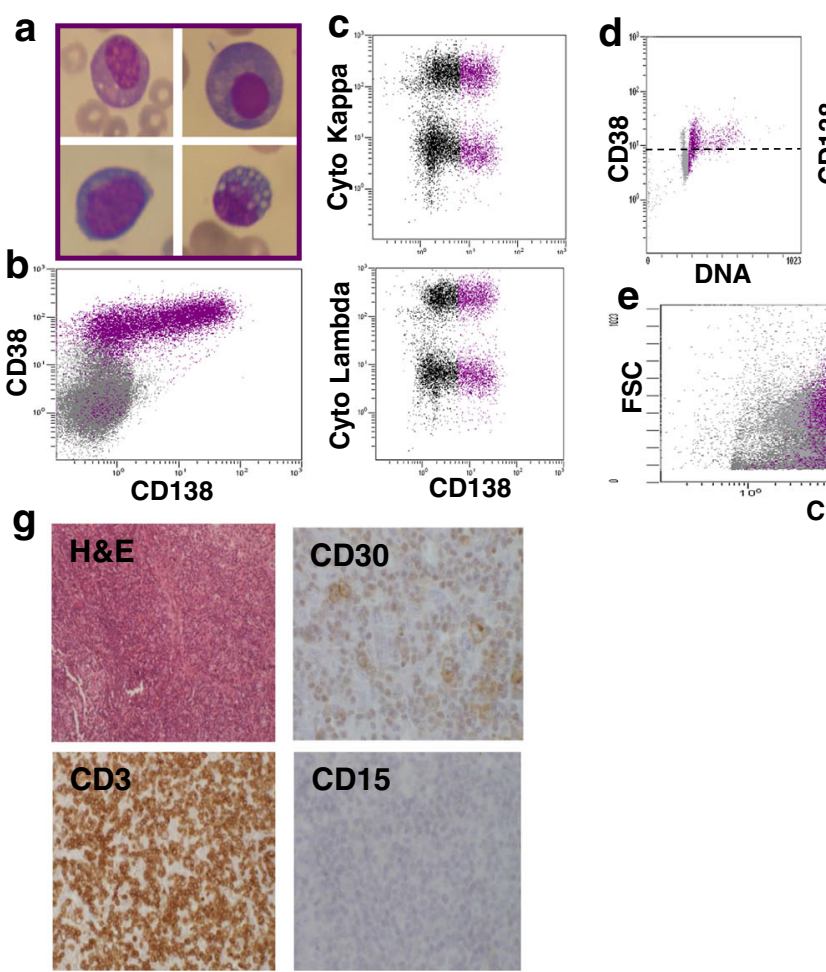
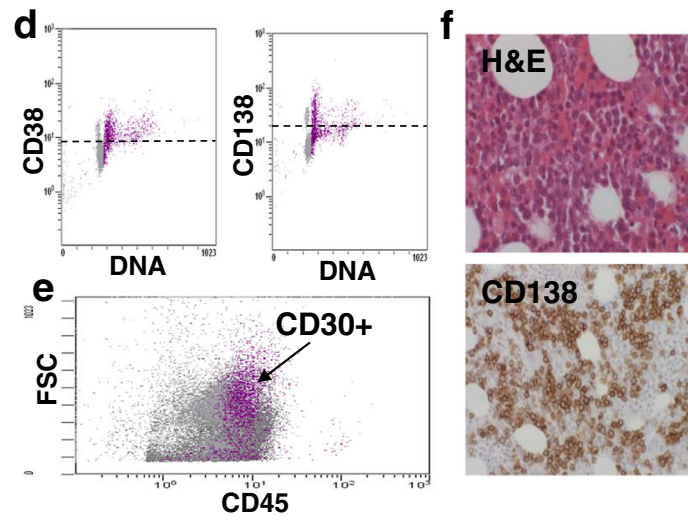

cell proliferation was confirmed by CD21 positivity which together with clonal T TCRB and TCRG rearrangement suggested the diagnosis of $\mathrm{T}$ cell lymphoma, most probably the angioimmunoblastic subtype (AITL).

Reactive plasmacytosis was previously defined as an expansion of plasmablasts retaining the capacity to differentiate into plasma cells [2]. Although reactive plasmacytosis has been described in different reactive and malignant conditions $[1,4]$, the relative amount of reactive PCs that we found in our case (20\% in PB and $30-40 \%$ in BM) is remarkable. We assume that the pattern of variable CD138 expression we found in our case represents a spectrum of PCs with different maturation stages. The possibility that this variable CD138 represents loss of expression in apoptotic PCs seems unlikely as we did not detect an increase of apoptotic cells by the sub G0-G1 area of the DNA analysis we performed. Among the different immunophenotyping characteristics of the PCs, the non-restricted cytoplasmic Igs demonstrated was the most useful tool for determining reactive plasmacytosis in our case. In our case, we detected clonal IgH rearrangement in the BM and polyclonal IgH rearrangement in the nodal specimen. Clonal IgH rearrangement was reported in up to $40 \%$ of AITL cases and suggested to be the result of the B cell expansion observed in the disease [1]. Accordingly, the clonal $\operatorname{IgH}$ rearrangement in the BM may be explained by the massive plasmablasts expansion at this site. Lack of equivalent clonal IgH rearrangement in the nodal specimen may be explained by the possibility that the plasmablasts are less expended at this site. However, we also could not exclude the possibility that this inconsistency resulted from the poor quality of the nodal specimen.

HRS-like cells with a background of plasma cells were previously described in the context of T cell lymphomas [3]. The immunophenotypic findings of HRS-like cells in these cases demonstrated strong CD30 staining, focal positivity to $\mathrm{CD} 15$, and variable immunoreactivity to CD20. The finding of CD30+ cells suspected as HRS-like cells in our case by flow cytometry is currently anecdotal, and further characterizing these cells in other cases is needed to verify their diagnostic utilization.

\section{Compliance with ethical standards}

Funding This study has no funding.

Conflict of interest The authors declare that they have no competing interests.

Ethical approval All procedures performed in this study were in accordance with the ethical standards of the institutional and/or national research committee and with the 1964 Helsinki declaration and its later amendments or comparable ethical standards. 
Informed consent Informed consent was not obtained from the individual participant included in the study.

\section{References}

1. Huppmann AR, Roullet MR, Raffeld M, Jaffe ES (2013) Angioimmunoblastic T-cell lymphoma partially obscured by an Epstein-Barr virus-negative clonal plasma cell proliferation. J Clin Oncol 31(2):e28-e30
2. Jego G, Robillard N, Puthier D, Amiot M, Accard F, Pineau D, et al. (1999) Reactive plasmacytoses are expansions of plasmablasts retaining the capacity to differentiate into plasma cells. Blood 94(2):701-712

3. Nicolae A, Pittaluga S, Venkataraman G, Vijnovich-Baron A, Xi L, Raffeld M, et al. (2013) Peripheral T-cell lymphomas of follicular Thelper cell derivation with Hodgkin/Reed-Sternberg cells of B-cell lineage: both EBV-positive and EBV-negative variants exist. Am J Surg Pathol 37(6):816-826

4. Shtalrid M, Shvidel L, Vorst E (2003). Polyclonal reactive peripheral blood plasmacytosis mimic 